\title{
The Research on Mock Exam System Design for English Listening and Speaking \\ LING ShuangYing ${ }^{1, a}$
}

${ }^{1}$ Business English Department, Anhui Institute of International Business, Anhui, Hefei, 231131, China

alingshuangying@126.com

Keywords: Mock Exam; English Learning; Listening and Speaking

\begin{abstract}
With the arrival of information age, network technology, database technology, multimedia technology is widely used by people in all walks of life, the online examination system based on Internet technology and produce also is able to produce, it can obviously alleviate the burden of teaching, a precise feedback teaching effect, effectively save the test cost, and can reflect the objective, fair and open education concept. English simulation test system designed in this paper based on the J2EE multi-layer architecture, with functions of automatic grading and statistical analysis, the structure is very simple. Can achieve national English test all the major topic of automatic testing, and can automatically detect the speech errors and defects existing in the pronunciation, for large-scale learners and develop the English listening test of the improvement of English listening and speaking ability has the positive significance.
\end{abstract}

\section{Introduction}

With the arrival of information age, network technology, database technology, multimedia technology is widely used by people in all walks of life, education is a profound technological revolution broke out. Online training, emerging teaching methods constantly emerging, such as remote teaching based on Internet technology and the online examination system also is able to produce, it can obviously alleviate the burden of teaching, a precise feedback teaching effect, effectively save the test cost, and can reflect the objective, fair and open education concept [1]. Add heard in high school examination is to build one of the effective measures of this system, will be great help for building perfect education system, to cultivate adapted to the rapid development of economy and society "generalists" is very necessary, to meet the social demand for high-quality talent strategic significance [2].

Made a great development at the same time, the Internet and campus network, all kinds of English test has been widely used computer technology, the researchers abroad to take part in the speaking test the examinee to make use of the computer system pronunciation assessment. Domestic middle school English education is also actively develop English test system based on network. English listening and speaking in English teaching is an indispensable important link, is also an important way of language information and language feeling, listening teaching and training is more and more important. English simulation test system designed in this paper based on the J2EE, adopts B/S architecture, with functions of automatic grading and statistical analysis, the structure is very simple.

\section{J2EE development platform}

J2EE (Java2 Platform Enterprise Edition) is a practical industry standard using Java technology to develop enterprise applications, it is a Java technology constantly that adapt to and promote the product in the process of enterprise applications [3]. It provides a unified platform for the development, not only reduces the cost and complexity of developing multi-tier application, and accelerate the application of distributed deployment, increased security mechanism, improve the performance of the system. J2EE with its good cross-platform portability, multi-layer architecture system, security, scalability, load balancing and reusability and enterprise-class platform based on 
the standard features, to make it in today's software development occupied the leading position unshakable.

Based on J2EE platform application uses a multi-layer distributed application model, it according to the function, application logic can be divided into different components, each according to which they belong to different J2EE application component layer and be installed on different machines [4], as shown in figure 1.

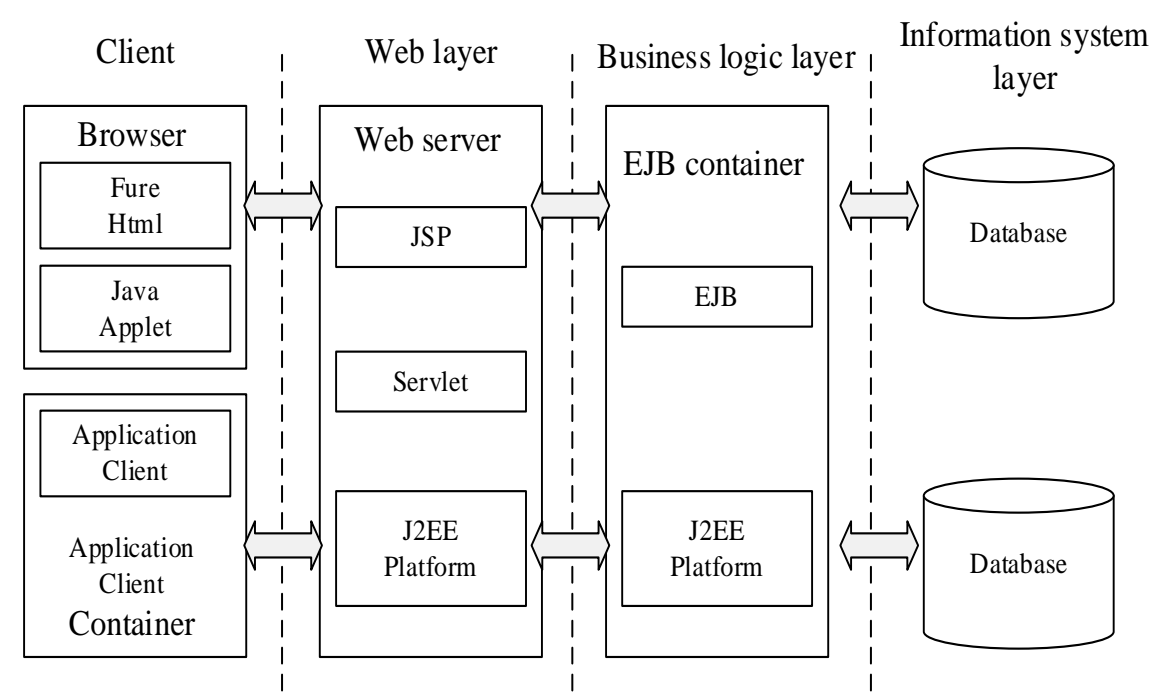

Figure 1. The structure of J2EE development platform

A multi-layer distributed application model means application logic according to the function and divided into components, and can be installed in the same server or on a different server of J2EE application of these different components. An application components should be installed where, depending on the application components belong to which layer of the multilayer J2EE environment. These layers is a customer layer, middle layer (including Web layer and business layer) and enterprise information system (EIS).

J2EE platform provides a component-based method, to design, development, assembly and deployment of enterprise applications. J2EE platform provides a multi-layer distributed application model, the security model of component reuse, consistent, and flexible transaction control. You can not only faster than ever before to market innovative customer solutions, and your platform independent, based on the J2EE solution components will not be binding on any one vendor products and API.

\section{The function analysis of the English mock exam system}

English simulation test system is in an age of computer technology and network technology rapid development background, design and development of the local area network (LAN) a computer classroom based on school exam system, it well embodies the contemporary junior middle school English teaching reform, a new concept. It not only can provide the whole simulation test environment, also can undertake where some unity, lighten the burden of teaching teachers reading scores, can also promote the improvement of students heard that [5].

After demand research, we believe that an ideal English simulation examination system should meet the following main functions: system easy to deploy within the local area network (LAN), the user group is clear, the control ability of information security and data manipulation and transaction processing ability is stronger, the server and client interaction effect is good. System can be divided into before, the most important function of stressed before and after the exam, system a high degree of automation, to realize the operation process and test process of synchronization, test and automatic grading students synchronization, generate assessment can be automated. System by administrators, teachers, students, three subsystem modules, and each module of the use of and 
access to different, but the mutual coordination between each other. According to the requirements described above, determine the function of the system summary is shown in figure 2 .

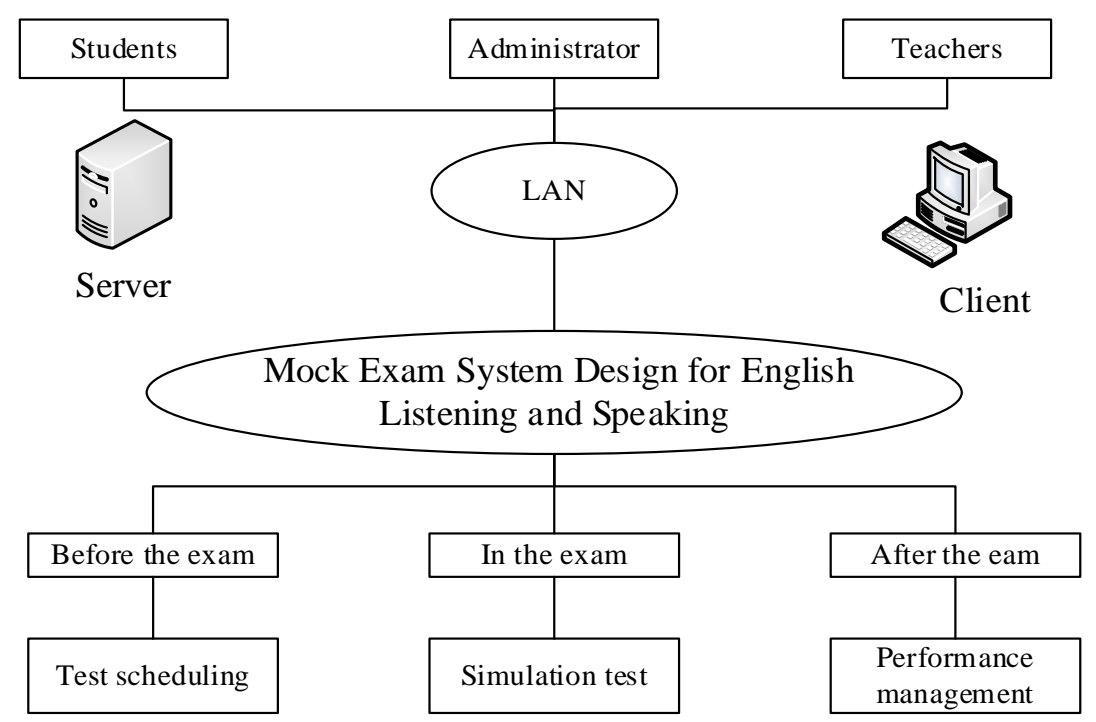

Figure 2. The function module of the English mock exam system

According to the above description of the test process, the whole system is divided into three subsystems: functionally arrangement system, examination system and achievement management subsystem. Choreography system management subsystem is responsible for the test questions and related information. Examination subsystem is responsible for the whole process of examination, including validation of examinee information, examination time, examination is terminated, automatic objective evaluation, subjective topic teacher evaluation and result output, etc.; System management is responsible for the personnel information, course information, the test information, information query, real-time monitoring, and the management of the performance statistics.

\section{English mock exam system overall design}

Software architecture is the basis of the constructing practice of computer software, it is a series of abstract patterns, used to guide the design of the aspects of large software systems. Software architecture is a sketch of the system. The software architecture description of object is directly constitute a system of abstract components. The connection between the various components are clear and relatively detailed description of the communication between components. In the implementation phase, these abstract components are divided into actual components, such as a specific class or object. In the field of object oriented, the connection between the components usually use interface to implement. According to the demand analysis of this system, combined with the current technology development present situation, when designing the system to adopt the J2EE multi-layer architecture design, detailed as shown in figure 3.

System adopts B/S structure of J2EE, concise structure. Each module interface using the WFP, the UI show more excellent. Statistics section with the method of embedded browser opens the page of the server implementation, using Ajax, JSON data interchange format, and use Java Script High charts show, compatible with all major browsers today, loading speed faster, and better performance effect. Data storage using lightweight SQLite database, deploy more flexible and convenient. Evaluating engine USES the latest SEE42 engine. At the same time, the system also exist deficiencies. First, the evaluation test paper module, easy to appear a lot of detail problems, many of which part need to APP interface. It needs to have a good understanding of simulation test process, and familiar with each table in the database and the connection between the each table. Again, in the simulation test module, machine configuration reasons such as easy to crash the IIS. In addition, the engines will also review abnormal problems, need many times to adjust mock exam system. 


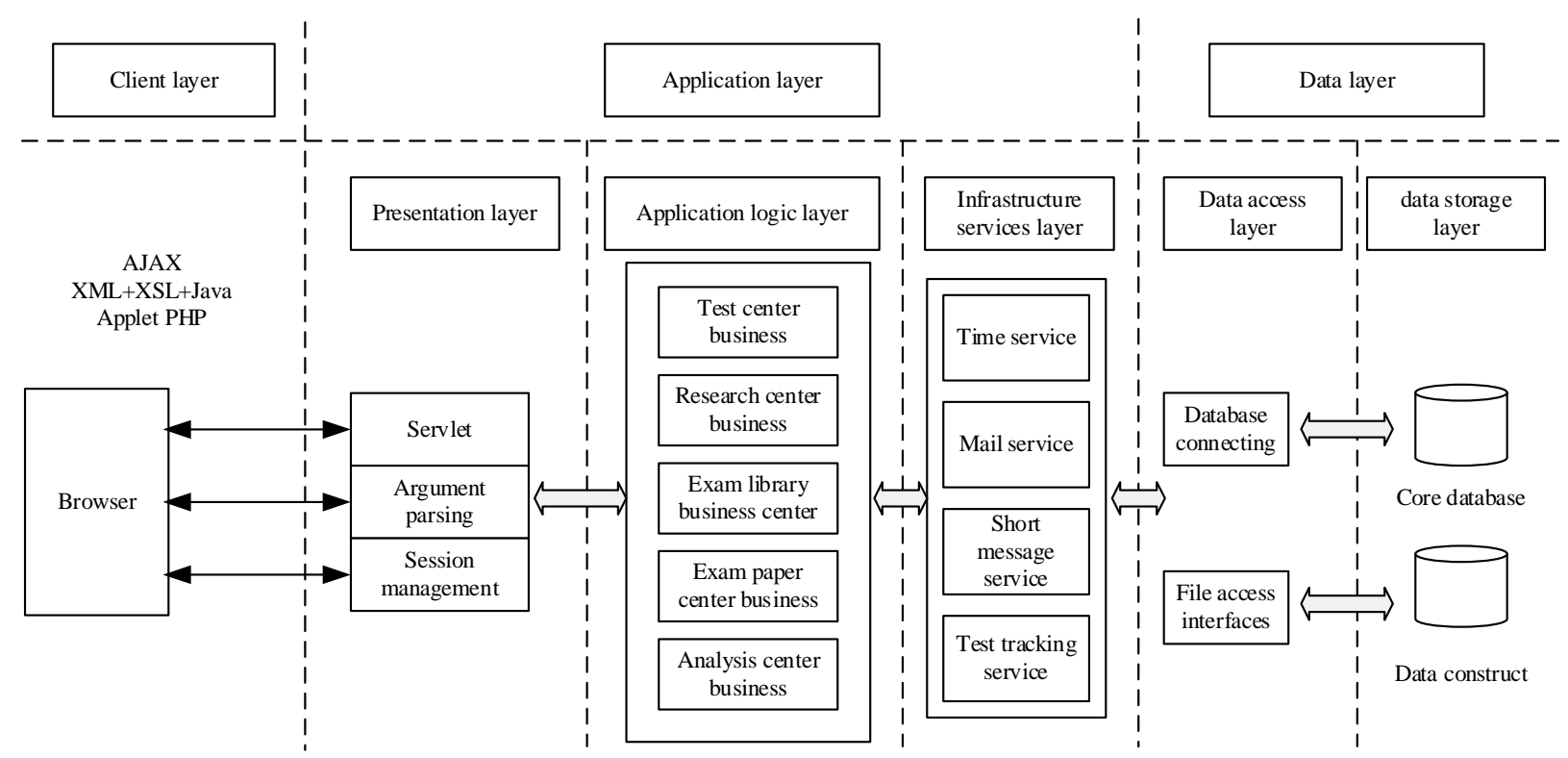

Figure 3. The structure of English mock exam system

Choose J2EE technology, can separate the system development for the customer layer, application layer, data layer. Client layer is designed, and the user interaction, through the browser interacts with the system. The logic of application layer is the main aspects of the implementation, the system test, investigation, analysis, paper and other business is done through this layer, the last is the data layer, including data access and data storage, such data can be independent. Through this architecture makes clearer the purpose of each layer, layer of abstraction degree is higher, higher development efficiency.

\section{Conclusion}

English simulation test system is according to the method of software engineering to carry on the design and implementation. This system is divided into three main modules: the examination management module, simulation test module and evaluation module. Between each function module process clear, clear hierarchy, can easily simulated test for students and teachers. The system uses the J2EE multi-layer architecture design, compact structure. As a system based on school classroom computer LAN, English simulation test system can not only provide the whole simulation test environment, also can be gained through the intelligent grading technology real-time heard scores, the teacher can carry on the examination paper evaluation according to the result of the score in the classroom, the reducing load teacher marking, raise the level of students heard that.

\section{Reference}

[1] T.Y. Liu, Y.L. Chu: Computers \& Education, Vol.55 (2010) No.2, p. 630.

[2] K. Zechner, D. Higgins, X. Xi: Speech Communication, Vol.51 (2009) No.10, p. 883.

[3] T.Y. Liu: Journal of Computer Assisted Learning, Vol.25 (2009) No.6, p. 515.

[4] A. Jonas, G. Chwo: Great Light Journal Vol.59 (2010) No.1, p. 25.

[5] G.J. Ockey: The Modern Language Journal, Vol.93 (2009) No.1, p. 836. 